\title{
Chaotic Optimal Operation of Hydropower Station with Ecology Consideration
}

\author{
Xianfeng Huang ${ }^{1}$, Guohua Fang ${ }^{1}$, Yuqin Gao ${ }^{1}$, Qianjin Dong ${ }^{2}$ \\ ${ }^{1}$ College of Water Conservancy and Hydropower, Hohai University, Nanjing, China \\ ${ }^{2}$ College of Water Resources and Hydropower, Wuhan University, Wuhan, China \\ E-mail:hxfhuang2005@163.com \\ Received April 21, 2010; revised June 2, 2010; accepted July 10, 2010
}

\begin{abstract}
Traditional optimal operation of hydropower station usually has two problems. One is that the optimal algorithm hasn't high efficiency, and the other is that the optimal operation model pays little attention to ecology. And with the development of electric power market, the generated benefit is concerned instead of generated energy. Based on the analysis of time-varying electricity price policy, an optimal operation model of hydropower station reservoir with ecology consideration is established. The model takes the maximum annual power generation benefit, the maximum output of the minimal output stage in the year and the minimum shortage of eco-environment demand as the objectives, and reservoir water quantity balance, reservoir storage capacity, reservoir discharge flow and hydropower station output and nonnegative variable as the constraints. To solve the optimal model, a chaotic optimization genetic algorithm which combines the ergodicity of chaos and the inversion property of genetic algorithm is exploited. An example is given, which shows that the proposed model and algorithm are scientific and feasible to deal with the optimal operation of hydropower station.
\end{abstract}

Keywords: Hydropower Station Operation, Ecology, Chaotic Genetic Optimization Algorithm, Time-Varying Electricity Price

\section{Introduction}

The optimal operation of hydropower station can increase the hydropower market competitive ability and realize the optimization of resources. How to manage and utilize the present hydropower station and to obtain the more comprehensive benefits in the case of maintain the extra investment, which have important significance on the development of our national economy and society and solve the problem of the energy shortage in short time. Investigation into the hydropower station optimal operation arises from the 1940s, in 1946, Masse the first introduced the concept of optimization into hydropower station optimal operation. American scholar Little [1] propose a random model of reservoir optimal operation which random variable is the runoff. Now, many scholars carried out earlier reservoir optimal operation of research and application [2-5]. At present hydropower station reservoir operation have more research on optimization algorithm and reached many great results. Many arithmetic have been applied to hydropower station reservoir optimal operation [6-13], such as dynamic programming, decomposition-coodination method of large systems, fuzzy mathematics, genetic algorithm, artificial neural network, particle swarm optimization algorithm, ant colony optimization algorithm. With the continuous development of theory and method for reservoir optimal operation and in-depth development of electricity market reform, the study of hydropower station reservoir optimal operation is a hotspot research within a period of time in the future. In addition, with the development of people's knowledge about the ecological environment, some scholars become to concern the ecology for optimal operation of hydropower station $[14,15]$. But most studies of the existing reservoir are also rarely considered the ecological water requirements of the river downstream and the reservoir itself. Although the models and algorithms have made considerable progress, but most of the models and the algorithms have bad universality, and is often for the specific reservoir conditions and operating characteristics which develop the specific model and algorithm. In this paper, based on the predecessors, under the conditions of researching the electricity market, reasonable consider the ecological water requirements, the chaotic optimal operation model based on time-varying electric- 
ity price is established, chaotic genetic arithmetic(CGA) is exploited to solve the model, the study has a certain reference practice value for optimal operation of hydropower station.

\section{Time-Varying Electricity Price}

In electric power market, competition system is introduced, and the policy of time-varying electricity price, such as flood and dry power price, peak and valley power price is carried out. The key of hydropower companies' business is to achieve the maximum power generation benefit considering the various constraints, through optimal arranging the generation process. Due to the characteristic of hydropower, such as the uncertainty and randomicity of runoff, the regulating ability of reservoir, the hydropower station reservoir optimal operation is much more complex. So, how to forecast the reservoir runoff, how to reasonably arrange generation operation to improve the economy benefit based on integrated utilization request and the regulating ability of reservoir and the flood and dry power price, become more and more important.

In electric power market, hydropower companies participate in market competition through declaring energy price curve. Because the policy of "plant and network separation, electricity price bidding" is carried out, the traditional optimal operation is challenged. The surroundings up against the hydropower plant have changed profoundly, and so the optimal objective of hydropower plant. Along with the independency of property right of hydropower companies, the maximum income and profit is pursued. The rule of maximum power generation energy in the past is substituted by the power generation benefit in the electric power market.

In the condition of electricity price bidding, the optimal operation of hydropower station reservoir considers not only the water quantity factor, but also the electricity price factor. At the view of market economy, the hydropower plants pay more attention to the timeliness of power generation energy and acquire more economic benefit.

In the past few years, especially in the summer, power consumption load has increased rapidly. Many provinces of China suffer the lack of electricity. Besides, people's life habit in a day makes the electricity load high in the day, and low in the night. The big difference of peak and valley usually causes some bad effects for power system, such as causing the operation difficulty, depressing the economy of the system. The best method to solve the problem is carrying out flood and dry power price and peak and valley power price. That is to say, the power price falls in the flood season, and increases in the dry season. It forms a net power price structure with price difference in different seasons. The peak and valley pow- er price includes peak load power price, valley load power price and smooth load power price which ascertained by the peak load period, valley load period and smooth load period in the daily load curve [16].

The time-varying electricity price can promote uses to avoid peak, and make the best of valley load. It can make the distribution of the power load curve uniform. In addition, at the view of consumer demand and provider benefit, the policy of time-varying electricity price can increase power energy sales, reduce generation cost and improve the system operation benefit. In the other hand, it can provide preferential electricity price, save the expense for uses. So it is favorable for consumer and provider. The one-part rate system price is fixed and unchangeable, and the income of hydropower companies is the product of generated energy and power price. That is to say, the maximum benefit is equal to the maximum of generated energy. But for the time-varying electricity price, the two are different. So the optimal operation based on time-varying electricity price is a new research problem for hydropower station reservoir.

\section{Optimal Operation Model with Ecology Consideration}

\subsection{Guidelines for Optimal Scheduling}

Optimal scheduling of hydropower station reservoir optimization operation is generally divided into two groups: the quantity and quality, including which makes the greatest economic benefits of electricity generation and makes the highest quality of electricity and water supply. From the economic point of view the biggest, in the absence of the implementation of power market, for hydropower station, the most common method is to make a maximum generating capacity in operation period. Under the environment of electricity market, the most important is to combine the electricity price, in accordance with peak and valley changes in policy, make reasonable arrangements for generation companies which owned the water and thermal power generation capacity at different times, and then the power companies can get the most economic benefits in operation period.

\subsection{Objective Functions}

In order to achieve the most optimal use of water resources, in this study, the goal is to meet the premise of water requirements, the criterion is to improve the power generation companies' earnings, and provide the greatest possible reliability and power to the grid, so, choose the following two objectives:

Objective I: Obtain the maximum annual power generation benefit per every year, by the reservoir regulation, 
so as to increase the profit of hydropower station in the dry season and flat-water period, and then increase revenue generation as much as possible in the wet period. The objective can be seen as follows:

$$
\max F=\sum_{t=1}^{T} A p_{t} Q_{t} H_{t} M_{t}
$$

where, $F$ is the effectiveness of the annual hydropower generation(RMB). $A$ is the comprehensive output coefficient of hydropower. $P_{t}$ is the power price factor; $Q_{t}$ is the generating flow of hydroelectric power on the time $t\left(\mathrm{~m}^{3} / \mathrm{s}\right) . \quad H_{t}$ is the average water head of hydroelectric power on the time $t(\mathrm{~m}) . T$ is the hydropower operation calculation of the total time. $M_{t}$ is the number of hours in the time $t$.

Objective II: Obtain the maximum output of the minimal output stage in the year. The objective can be seen as follows:

$$
\max N P=\max \min \left\{A Q_{t} H_{t}\right\}
$$

where, $N P$ is the maximization minimum output of hydropower station $(M W)$. Other symbols are the same as (1).

Objective III: Obtain the minimum amount of ecologyical water shortage for river downstream and the reservoir itself.

$$
Z=\min \sum_{\mathrm{t}=1}^{N}\left(R L_{(t)}+V L_{(t)}\right)
$$

where, $Z$ is the total ecological water shortage. $R L_{(t)}$ is the ecological water shortage of the river downstream at time $t$. $V L_{(t)}$ is the ecological water shortage of the reservoir itself at time $t . N$ is the total time.

Ecological water demand computing $[17,18]$. As the ecological water requirements of surviving in the debate, some scholars have raised the minimum ecological water and suitable ecological water demand, water demand of ecological environment in this article is based on the minimum water volume of ecological water demand on the basis of calculation. Therefore, (3) can be written as:

$$
Z=\min \sum_{\mathrm{t}=1}^{N}\left(R D_{(t)}+V D_{(t)}-R S_{(t)}-V S_{(t)}\right)
$$

where, $R D_{(t)}$ and $V D_{(t)}$ are the minimum amount of ecological water demand of the river downstream and reservoir itself respectively. $R S_{(t)}$ and $V S_{(t)}$ are the supply amount of ecological water of the river downstream and reservoir itself respectively.

The traditional optimal operation of hydropower reservoir main considers socio-economic objective, with little regard the requirements of the ecological environment, leading to environmental degradation. The objective of minimum ecological water shortage of river downstream and reservoir fully reflects the lower reaches of the reservoir and river ecology to environmental protection, so that human life and the ecological environment has been the basic water was placed in the same the degree of importance for the protection of river health, and promote sustainable utilization of water resources play an important role.

\subsection{Constraints}

1) Reservoir water balance constraint.

$$
V_{t+1}=V_{t}+\left(q_{t}-Q_{t}\right) K_{t}
$$

where, $V_{t+1}$ is the reservoir storage capacity in the end of time $\mathrm{t}\left(\mathrm{m}^{3}\right) ; V_{t}$ is the reservoir storage capacity in the beginning of time $t\left(\mathrm{~m}^{3}\right) ; q_{t}$ is the average inbound flux on the time $t\left(\mathrm{~m}^{3} / \mathrm{s}\right) ; K_{t}$ is the conversion factor of the time length.

2) Reservoir storage capacity constraint. At any time, the reservoir water storage capacity storage capacity should be kept between a minimum and maximum volume of water.

$$
V_{t, \text { min }} \leq V_{t} \leq V_{t, \max }
$$

where, $V_{t, \min }$ is the allowed minimum reservoir capacity on the time $t\left(\mathrm{~m}^{3}\right) . V_{t, \max }$ is the allowed maximum reservoirs capacity on the time $t\left(\mathrm{~m}^{3}\right)$.

3) Reservoir discharged flow constraint.

$$
Q_{t, \min } \leq Q_{t} \leq Q_{t, \max }
$$

where, $Q_{t, \min }$ is the minimum discharge on the time $t$ $\left(\mathrm{m}^{3} / \mathrm{s}\right) ; Q_{t, \max }$ is the maximum discharge on the time $t$ $\left(\mathrm{m}^{3} / \mathrm{s}\right)$.

4) Hydropower station output constraint.

$$
N_{t, \text { min }} \leq A Q_{t} H_{t} \leq N_{t, \text { max }}
$$

where, $N_{t, \text { min }}$ is the minimum output which hydropower station allowed, it is always the guaranteed output (kW). $N_{t, \text { max }}$ is the maximum output which hydropower station allowed, it is always the installed capacity $(\mathrm{kW})$.

5) Nonnegative variable constraint.

All of the above decision variables are non-negative variables $(\geqslant 0)$.

\section{Chaotic Genetic Algorithm}

\subsection{Thought of Chaotic Genetic Algorithm}

Chaos is a seemingly rule, similar to the random phenolmenon which emerge in deterministic system, the theo- 
ries marked by the United States meteorologist Lorenz in 1963, which published papers named "Deterministic non-periodic flow" [19], this paper reveals the existence of deterministic chaos in nonlinear equation. Nowadays, scholars generally agreed that chaos with such features, include randomness, regularity, and ergodicity [20], due to chaotic motion can after all states according to their own laws within a certain range of non-repetition. Therefore, use chaotic variables to optimization search, certainly the global optimal solution can be obtained and have high search efficiency. At present, the chaos optimization have been applied in optimal operation of reservoirs [21, 22], on the basis of previous research, this paper coupled the chaos optimization and genetic algorithms, combined with multi-objective decision-making techniques to develop the chaotic genetic algorithm (CGA). The first, CGA use constraint method converted multi-objective problem to single objective problem, using penalty function method dealing with constraints. And then chaotic variables were introduced into the optimization variables, and to enlarge the scope of chaotic motion to the range of optimization variables, code chaotic variables which we got, using the search mechanisms of genetic algorithm to obtain the optimal solution. The basic idea of CGA solving hydropower station reservoir optimal scheduling is: the first, scheduling period (usually one year) is divided into a number of time slots $T$, numbered the sequence numbers of each period, choose every period of the reservoir water level value (the value of reservoir storage capacity can also be used) as optimization variables, determine the upper and lower limits of the reservoir water level value each time, randomly selected $\mathrm{n}$ different initial values which interval is between 0 and 1 , through Logistic maps can obtain the n-chaotic trajectories of different sequence, the length of the chaotic sequence is the population size, large it to the range of reservoir water level at all times, then get $\mathrm{n}$ group sequence of the reservoir water level which stand forthe reservoir operation control process $\left(Z_{1}^{1}, Z_{2}^{1}, Z_{3}^{1}, \cdots, Z_{T}^{1}\right),\left(Z_{1}^{2}, Z_{2}^{2}, Z_{3}^{2}\right.$, $\left.\cdots, Z_{T}^{2}\right), \ldots,\left(Z_{1}^{n}, Z_{2}^{n}, Z_{3}^{n}, \cdots, Z_{T}^{n}\right)$, and as a mother, according to the intended target function evaluate its advantages and disadvantages, calculate the fitness value of all chromosomes, carry out selection, crossover and mutation operations according to the chromosomes fitness, use the most excellent retention strategy, abandon the low fitness chromosomes, to retain the high fitness chromosomes, and thus get new groups. Add a chaotic small perturbation to the optimization variables, by evolving from generation to generation, then finally converge to one individual which in the most suitable environment, and obtain optimal solution to the problem.

\subsection{Multi-Objective Decision}

The basic springboard of chaotic optimization is the er- godicity, that chaotic motion can pass all states nonrecurring in a certain range. The characteristic can be effective mechanism of avoiding local optimal solution and the difficulty of the continuity and differentiability of objective functions and constraints. The idea of chaotic optimization is to transfer area coverage from chaotic series to decision variables, use the new chaotic variables for searching and iterative comparison. If the criterion of stop is satisfied, then export the optimal results. Mathematically, the general multi-objective constrained optimization problem can be stated as follows:

$$
\begin{array}{ll}
\min & \boldsymbol{y}=f(\boldsymbol{x})=\left\{f_{1}(\boldsymbol{x}), f_{2}(\boldsymbol{x}), \cdots, f_{m}(\boldsymbol{x})\right\} \\
\text { s.t. } & g_{i}(\boldsymbol{x}) \leq 0 \quad i=1,2, \cdots, k \\
& h_{j}(\boldsymbol{x})=0 \quad j=1,2, \cdots, l
\end{array}
$$

where, $\boldsymbol{x}$ is the decision vector. $\boldsymbol{x}=\left(x_{1}, x_{2}, \cdots, x_{n}\right)$ $\in \boldsymbol{X} \subset R^{n} . \boldsymbol{X}$ is decision space. $\boldsymbol{y}$ is the objective function vector. $\boldsymbol{y}=\left(y_{1}, y_{2}, \cdots, y_{m}\right) \in \boldsymbol{Y} \subset R^{m} . \quad \boldsymbol{Y}$ is objective space. $f(\boldsymbol{x})$ is the objective function to be optimized, $g_{i}(\boldsymbol{x}), h_{j}(\boldsymbol{x})$ are the constraints imposed on the design, $n, m(m>1)$ are the dimensions of decision vector and objective functions respectively.

For multi-objective optimization problem, it is difficult to find absolute optimal solution. Mostly, we choose the best equilibrium solution which has precision to a certain extent and practical significance according to the request of problem.

Traditional multi-objective programming method is linear weighted sum method. It converts the problem to single objective problem by weighted coefficient. There are some disadvantages such as the units of different objectives are not the same for comparison and subjectivity is obvious. This paper adopts constraint method. Supposing the problem has $p$ objectives. The idea is to ascertain a main objective $f_{1}(x)$, and take the $p-1 \mathrm{ob}-$ jectives as secondary targets, choose some threshold values $u_{j}(j=2,3, \cdots, p)$ through the experience of decision-makers, then change the secondary targets to constraints. So the problem is to solve the single objective optimal problem as follows.

$$
\begin{aligned}
& \min f_{1}(x) \\
& \text { s.t. } x \in \tilde{S}=\left\{x \in S \mid f_{j}(x) \leq u_{j}, j=2,3, \cdots, p\right\}
\end{aligned}
$$

\subsection{Constraints Treatment}

Chaotic optimization is a direct search method, which requests dealing with the constraints. Penalty function method is effective for the constraints treatment. Its basic idea is to add a penalty item to the objective function as 
(11), converting the initial problem to the new non-restraint optimization problem with the penal function, washing out the non-feasibility solution through punishing the solutions dissatisfying the constraints and finally gaining the optimal solution.

$$
p(\boldsymbol{x}, \sigma)=f(\boldsymbol{x})+\sigma(c(\boldsymbol{x}))
$$

where, $f(\boldsymbol{x})$ is the objective function of initial problem, $\sigma(c(\boldsymbol{x}))$ is the penalty item.

The paper adopts non-differentiable exact penalty function method to convert the constraints to non-restraint optimization problem. It avoids the sequence character in computation, accords the solution of restraint optimization with the minimum points of penalty function. It also avoids the difficulty of the non-differentiability, which is effective for the optimization without grads information. With the work upwards, multi-objective restraint optimization problem is converted to single objective non-restraint optimization problem.

In the paper, objective II and objective III is transferred into constraints by non-differentiable exact penalty function method. The specific action is: firstly, amplification to guaranteed output, as lower limit output of the minimum time output. The value needs several trials. Secondly, the minimum ecological water shortage objective will be transferred to two constraints, one is to meet the minimum ecological water demand of river downstream, and the other is to meet the minimum ecological water demand of the reservoir itself.

The objective II can be transferred to guaranteed output constraints as follows:

$$
A Q_{t} H_{t} \geq N_{0}
$$

where, $N_{0}$ is the amplified guaranteed output.

The objective III can be transferred to ecological water demand constraints. Considering the ecological hydropower station reservoir operation, the reservoir and downstream of the ecological and environmental problems were emphatically solved, the following river ecological water requirements lower bound to determine the process of ecological environment of the reservoir discharged. Therefore, the ecological water demand constraints including two aspects: ecological storage capacity constraints and the downstream of ecological water demand constraints.

$$
\begin{gathered}
\max \left(V L_{t}, V_{z e, t}\right) \leq V_{t} \leq V H_{t} \\
W h_{t} \geq W h_{t \min }
\end{gathered}
$$

where, $V_{z e, t}$ is the ecological environment storage capacity of reservoir; $V L_{t}$ is the dead storage capacity of reservoir; $V H_{t}$ is the beneficial capacity in non-flood period, in flood period is the flood control storage capacity; $W h_{t}$ is the discharged ecological flow of reservoir in time t period; $W h_{l \min }$ is the minimum ecological water demand of downstream in time $t$ period.

\subsection{Steps of CGA}

The idea of multi-objective chaotic genetic optimization algorithm is to decompose the problem into a single unconstrained optimization problem. Chaotic optimization theory and genetic algorithm are coupled to solve the optimization problem. The steps of CGA are as follows:

Step 1. Multi-objective decision. Constraints method is used to deal with the multi-objective problem. The problem is transformed into single problem by (11).

Step 2. Constraints treatment. Non-differentiable exact penalty function method is used to deal with the constraints. We choose a certain penal factor to constitute penalty item. The problem is converted into the non-restraint optimization problem according to (15). Then we gain the optimization problem of continuous object as follows:

$$
\min f\left(x_{1}, x_{2}, \cdots, x_{n}\right) \quad x_{i} \in\left[a_{i}, b_{i}\right] i=1,2, \cdots, n
$$

Step 3. Parameters setting. Ascertaining the numbers of variables is $n$, and the bounds is $\left[a_{i}, b_{i}\right]$, the population scale of genetic arithmetic is $\mathrm{M}$, the maximal iteration times of the arithmetic is $T$, the cross probability of crossover probability is $P_{c}$, the mutation probability is $P_{m}$.

Step 4. Initialization. Choosing $n$ different initial values and acquiring $n$ chaotic variable serial $\varepsilon_{i, p}$ through Logistic mapping. $i=1,2, \cdots, n, p$ is the length of the chaotic variable serial. Logistic mapping is as follows:

$$
\varepsilon_{i, j+1}=\mu \varepsilon_{i, j}\left(1-\varepsilon_{i, j}\right), \quad j=0,1,2, \cdots, p-1
$$

where, $\mu$ is controlled parameter, if $0 \leq \varepsilon_{0} \leq 1$, $\mu=4$, then the (11) is in chaotic status and has all characteristic of chaotic motion.

Step 5. Magnify the ranges of chaotic series into the confines of optimal variables with (17).

$$
x_{i, j}=a_{i}+\left(b_{i}-a_{i}\right) \varepsilon_{i, j}, \quad j=1,2, \cdots, p
$$

Step 6. Fitness function values calculation. Choose a proper fitness function to calculate the fitness value. Fitness value will be sorted in descending, select the 10 percent group on better fitness directly into the next generation of groups, all populations of the selection, crossover and mutation were carried out.

Step 7. Calculate the new fitness value and make adjustments, and sort the group according to the fitness value, then replace the worst of which $10 \%$ of fitness, and maintain the files. Calculate the average fitness value and compare with the maximum, if within the allowable error, then end the searching process, output the optimal solution, otherwise continue. 
Step 8. Plus a chaotic disturbance to the current generation of group fitness value less than 90 percent of the corresponding optimization variables, through the carrier means mapped to optimization variables, perform iterative calculation, with the increase of iterations number, iteration gradually approach to the optimal solution. Until the time, two figures out the difference between the average fitness is less than a pre-given small positive number. Chaotic disturbance can be done as follows: the number of iterations to meet the initial optimal solution $\left(x_{1}^{*}, x_{2}^{*}, \cdots, x_{n}^{*}\right)$ is mapped to $(0,1)$ interval, the optimal decision by the initial vector, denoted by $\delta^{\prime}$, the chaotic mapping function iteration times $K$ get chaotic sequence ( $K$ is the length of the chaotic sequence), set $\varepsilon_{k}$ in the chaotic sequence of the $k$ value $(k=1,2, \cdots, K)$, write $\varepsilon_{k}$ composed of the ground $n$-vector, by Equation (18) Find the Chaos decision vector $\delta_{k}^{\prime}$ after disturbance

$$
\delta_{k}^{\prime}=(1-\alpha) \delta^{\prime}+\alpha \delta_{k} \quad(k=1,2, \cdots, K)
$$

The formula $\alpha$ for the $(0,1)$, a value range can be adaptive selection, $\alpha$ search the initial large, late small, according to (19) to determine $\alpha$ :

$$
\alpha=1-\left(\frac{k-1}{k}\right)^{m}
$$

The formula $m$ for a positive integer, determined according to the number of objective function, generally greater than or equal to $2 ; k$ for the chaotic map iterations.

Step 9. According to the fitness value, resort the groups, calculate the average fitness and compare it with the maximum. If it is in the allowable error, end the process of optimization, and output the optimal solution, otherwise, go to Step 5 .

\section{Case Study}

Study a certain hydropower station data as an example.
The curve between reservoir capacity and water level upstream and the curve between water levels downstream with discharge of the hydropower station reservoir are given. The total reservoir capacity is 896 million $\mathrm{m}^{3}$, regulating capacity is 445 million $\mathrm{m}^{3}$, the normal water level is $977.0 \mathrm{~m}$, dead water level is $948 \mathrm{~m}$, flood-control water level is $966.0 \mathrm{~m}$. The coefficient of output power takes 8.3 , guaranteed output is $185 \mathrm{MW}$. Installed capacity is $1080 \mathrm{MW}$. The largest discharge is $1000 \mathrm{~m}^{3} / \mathrm{s}$. According to the time-sharing surfing electricity price policy, in dry season period on the basis of the flat water floating upward 50 percent, in flood period, it falls 25 percent on the basis of the flat water. In flat-water period, the benchmark price is $0.247 \mathrm{RMB} / \mathrm{kW} \cdot \mathrm{h}$, in dry season the floating price coefficient (December-next April) is 1.50 , in flat-water period (May and November) is 1.00, and in flood period (June-October) 0.75. In a day, the power price of normal period is carried out by regulation, spike and peak hours floating upward 22 percent, and valley hours fall 40 percent. According to the annual average runoff data, the fore-mentioned model and CGA is used for optimal operation. The initial population of the model takes 2000. Crossover probability takes 0.9 . Mutation probability takes 0.1 . Permitted error takes 1.0 $\times 10^{-8}$. The largest iteration number takes 50. Logistic mapping initial value takes the values belong to $[0.51$, 074]. The software of MATLAB is used for calculation. The program runs 10 times, and we take the best result of them. In each program runs, the results of each generation to be superior than the previous generation, or the results equal to the previous generation, it is the result of using the optimal retention policies. The final results can be seen in Table 1.

The ecological water demand of the river downstream is calculated by Tennant method and minimum monthly runoff method, and takes the bigger. The ecological water demand of the reservoir itself is less than dead storage reservoirs 451 million $\mathrm{m}^{3}$, so the smallest ecological water demand of the reservoir itself is met.

Table 1. The result of optimal operation of hydropower station in electricity market environment.

\begin{tabular}{ccccccc}
\hline Month & Initial level $/ \mathrm{m}$ & Final level $/ \mathrm{m}$ & Generating flow $/ \mathrm{m}^{3} / \mathrm{s}$ & Output $/ 10^{4} \mathrm{Kw}$ & Generated energy $/ 10^{8} \mathrm{Kw} \cdot \mathrm{h}$ & Generated benefit $/ 10^{8} \mathrm{RMB}$ \\
\hline 7 & 976.47 & 966 & 945.48 & 53.03 & 3.87 & 1.43 \\
8 & 966 & 948 & 1273.16 & 55.59 & 4.06 & 1.50 \\
9 & 948 & 948 & 1254.29 & 45.44 & 3.32 & 1.23 \\
10 & 948 & 976.56 & 766.30 & 37.62 & 2.75 & 1.02 \\
11 & 976.56 & 976.97 & 535.05 & 33.03 & 2.41 & 0.60 \\
12 & 976.97 & 976.79 & 330.70 & 20.65 & 1.51 & 0.28 \\
1 & 976.79 & 976.65 & 343.55 & 21.39 & 1.56 & 0.29 \\
2 & 976.65 & 976.91 & 382.60 & 23.80 & 1.74 & 0.32 \\
3 & 976.91 & 976.58 & 601.90 & 37.04 & 2.70 & 0.50 \\
4 & 976.58 & 979.96 & 493.82 & 31.17 & 2.28 & 0.42 \\
5 & 979.96 & 976.53 & 322.40 & 20.50 & 1.50 & 0.37 \\
6 & 976.53 & 976.47 & 337.15 & 20.94 & 1.53 & 0.57 \\
Total & & & & 29.21 & 8.53 \\
\hline
\end{tabular}


From Table 1, the initial and the final water level are meeting the upper and lower limits. The output value of each month is also within the control range. Generating flows of each month are also meeting the ecological water demand of flow downstream. Therefore, the results meet the requirements.

In order to illustrate the superiority of the algorithm, four kinds of methods including dynamic programming (DP), genetic algorithm (GA) and chaos optimization algorithm (COA) were used in this paper, use the four kinds of methods to calculate the hydroelectric reservoir optimal operation model, the results can be seen in Table 2 .

From Table 2, CGA has the best results, next are the GA and the COA, and finally is the DP. DP needs the state discrete-time and stores state information in the optimization. The more discrete points it has, the higher precision it gets. But it increases the optimization run-time very much. GA depends on random variables, and it does not need the storage of eliminated discrete points. Therefore, it shows an obvious superiority in solving these optimization problems. So for the same problems, GA can greatly save the computer's memory demand. However, because of the probability search features, the results of GA are unstable. COA maps the chaotic sequences which generate by Logistic mapping to the range of optimal variables, and then does iterative searching, fully using the ergodicity characteristic of chaotic optimization. The annual generating capacity which is calculated by COA is greater than that of DP, but it needs larger chaotic sequence length, the time consuming of the program is longer. Therefore, the search efficiency of COA needs to be improved.

CGA combines the advantages of GA and COA, using the ergodicity feature of chaotic optimization, mapping the chaotic sequences which generated by Logistic mapping to the ranges of optimization variables, and then using the optimization mechanism of genetic algorithm for selection. After crossover and mutation, a chaotic disturbance is added to the variables corresponding to the degree of optimization variables in the current generation of groups, and then carrying out the genetic manipulation until the termination of the proceedings meets the conditions. Finally, output the optimal solution. Therefore, CGA has the advantages of high efficiency, good convergence performance and it approaches to the global optimal solution better. So it is the best algorithm to solve the optimal operation of hydropower station reservoir. Of course, due to the need of larger population size to achieve ergodicity, the calculation time of CGA is longer than GA.

The paper also does the research that not considering the ecology. The objectives take the largest generated benefit and the maximum output of the minimal output stage in the year.

The generated energy of hydropower reservoir optimal operation is 8.62 billion RMB without ecology consideration, which is 9 million RMB more than that with ecology consideration. But in January and February, the generating flow is less than the minimum ecological water flows. The results can be seen in Table 3 .

Can be seen from Table 3, without considering the ecological optimal operation of hydropower generating capacity of the reservoir and consider the ecological results of optimal operation of hydropower station reservoir compared to an increase of only 0.09 million RMB about $1.06 \%$, the two are close, But do not take into account the environment of the river hydropower reservoir optimal operation of the negative environmental impact is far-reaching. Optimal operation of hydropower and ecological considerations will help protect the river environment and promote sustainable use of water resources; sacrifice in exchange for a small part of the power generation efficiency and harmonious social and economic development and ecological environment is worth it and very necessary.

\section{Conclusions}

In electric power market, as a result of "separate the station and network, price bidding", and the independent property of all generating companies, the focus of optimal operation for the power plant transforms from single safety production to taking economic benefits as central of all-round integrated development. Power generation benefit will be paid more attention by generation companies. In this paper, a multi-objective chaotic optimal operation model based on time-varying electricity price is established in electric power market, considering the ecological water requirements of downstream. To solve the model, a multi-objective chaotic genetic optimization algorithm is exploited, which has the advantages of high search efficiency, good convergence performance, faster pace converge to the global optimal solution. It greatly increases the efficiency and effectiveness of optimal operation, and enriches and develops the theory and method of optimal operation for hydropower station reservoir.

Table 2. Results calculated by different algorithms.

\begin{tabular}{cccc}
\hline Algorithms & $\begin{array}{c}\text { Optimal results } \\
/ 10^{8} \mathrm{RMB}\end{array}$ & $\begin{array}{c}\text { Increase the } \\
\text { proportion of annual } \\
\text { electricity output } / \%\end{array}$ & $\begin{array}{c}\text { Time } \\
\text { consuming } \\
/ \mathrm{s}\end{array}$ \\
\hline DP & 8.24 & 1.10 & 10.4 \\
GA & 8.51 & 3.86 & 54.9 \\
COA & 8.39 & 2.74 & 387.3 \\
CGA & 8.53 & 6.23 & 265.2 \\
\hline
\end{tabular}

Table 3. Results with and without ecology consideration.

\begin{tabular}{cccc}
\hline $\begin{array}{c}\text { With ecology } \\
\text { Consideration } \\
/ 10^{8} \mathrm{RMB}\end{array}$ & $\begin{array}{c}\text { Without ecology } \\
\text { consideration } \\
/ 10^{8} \mathrm{RMB}\end{array}$ & $\begin{array}{c}\text { Generated } \\
\text { benefit decrease } \\
/ 10^{8} \mathrm{RMB}\end{array}$ & $\begin{array}{c}\text { Decrease } \\
\text { ratio } / \%\end{array}$ \\
\hline 8.53 & 8.62 & 0.09 & 1.06 \\
\hline
\end{tabular}


As the development of people's consciousness about the ecological environment and the idea of harmonious coexistence between human and nature, the ecology is necessary in optimal operation of hydropower stations. The paper takes full consideration of the ecology water demand of the river downstream and the reservoirs itself. In the actual calculations, the objective is solved through the constraint method. The example shows that the result without ecology consideration is a little more than that with ecology consideration, but the negative environmental impact is far-reaching.

\section{Acknowledgements}

The authors wish to thank Yinqin CHEN of Nanjing University of Technology for her careful review and constructive modifications. And the work is supported by the The National Natural Science Fund of China (No. 50909073) and Natural Science Fund of Hohai University (No.2009422011) and the Fundamental Research Funds for the Central Universities (2013B1020084).

\section{References}

[1] J. D. C. Little, "The Use of Storage Water in a Hydroelectric System," Operational Research, Vol. 3, No. 2, March 1955, pp. 187-197.

[2] Y. C. Zhang, F. S. Li, Y. F. Du, Y. F. Huang and S. Y. Xiong, "The Optimization of Hydropower Station Reservoir Dispatching," in Chinese, Journal of Huazhong University of Science and Technology, Vol. 9, No. 6, December 1981, pp. 49-56.

[3] W. Y. Tan, S. X. Huang, J. M. Liu and S. X. Fang, “Application of Dynamic Programming in Optimizing the Regulation of Reservoirs of Hydroelectric Stations," in Chinese, Journal of Hydraulic Engineering, Vol. 13, No. 135, July 1982, pp. 1-7.

[4] Q. Huang and Z. Q. Yan, “A Discussion on Methods of Long-Term Optimal Operation of Hydropower Stations Reservoir," in Chinese, Journal of Hydro Electric Power, Vol. 3, No. 3, March 1987, pp. 1-7.

[5] G. W. Ma, "Study on Stochastic Optimum Problems for Multireservoir Hydroelectric Systems," in Chinese, Journal of Xi'an University of Technology, Vol. 7, No. 4, December 1988, pp. 65-74.

[6] D. G. Shao, X. Y. Song, J. Xia and Z. Q. Sun, "Research on Real-Time Optimal Flood Dispatching Model for Yanghe Reservoir," in Chinese, Advances in Water Science, Vol. 10, No. 2, February 1999, pp. 135-139.

[7] J. Wan and H. Y. Chen, "A Study on Optimal Operation of Hydropower Group by Combined Model of Decomposition Coordination and Aggregation-Decomposition of Large System," in Chinese, Journal of Hydroelectric Engineering, Vol. 15, No. 2, February 1996, pp. 41-50.

[8] S. Y. Chen and H. C. Zhou, "Fuzzy Optimal Decision Theory and Model of the Multistage and Multiobjective
System," in Chinese, Water Resources and Power, Vol. 9, No. 1, January 1991, pp. 9-17.

[9] W. Robin and S. Mohd, "Evaluation of Genetic Algorithms for Optimal Reservior Resources," Journal of Water Resource Planning and Management, Vol. 125, No. 1, January 1999, pp. 25-33.

[10] G. W. Ma and L. Wang, "Application of a Genetic Algorithm to Optimal Operation of Hydropower Station," in Chinese, Advances in Water Science, Vol. 8, No. 3, March 1997, pp. 275-280.

[11] T. S. Hu, Y. H. Wan and S. Y. Feng, "Research on the Artificial Neural Network Methodology for Multi-Reservoir Operating Rules," in Chinese, Advances in Water Science, Vol. 6, No. 1, January 1991, pp. 9-17.

[12] S. H. Zhang, Q. Huang, H. S. Wu and J. X. Yang, “A Modified Particle Swarm Optimizer for Optimal Operation of Hydropower Station," in Chinese, Journal of Hydroelectric Engineering, Vol. 26, No. 1, January 1996, pp. 41-50.

[13] G. Xu and G. W. Ma, "Optimal Operation of Cascade Hydropower Stations Based on Ant Colony Algorithm," in Chinese, Journal of Hydroelectric Engineering, Vol. 24, No. 5, May 1991, pp. 9-17.

[14] L. S. Suo, “Water Conservancy's 'Special Features'The New Ideas about the Water Conservancy Construction," in Chinese, China Water Resources, Vol. 38, No. 1, January 2003, pp. 25-26.

[15] Z. R. Dong, D. Y. Sun and J. Y. Zhao, "Multi-Objective Ecological Operation of Reservoirs," in Chinese, Water Resources and Hydropower Engineering, Vol. 38, No. 1, 2007, pp. 28-32.

[16] G. W. Ma, L. Wang and X. M. Guo, "A Optimal Operation Model for Multiple Reservoir Power with TimeVarying Electricity Price," in Chinese, Advances in Water Science, Vol. 13, No. 5, May 2002, pp. 583-587.

[17] H. P. Hu, D. F. Liu and F. Q. Tian, “A Method of Ecological Reservoir Reoperation Based-on Ecological Flow Regime," in Chinese, Advances in Water Science, Vol. 19, No. 3, 2008, pp. 325-332.

[18] W. G. Yu, Z. Q. Xia and G. R. Yu, "Analysis of Ecological Ideas and Measures in Reservoir Scheduling," in Chinese, Journal of Shangqiu Teachers College, Vol. 22, No. 5, 2006, pp. 148-151.

[19] E. N. Lorenz, "Deterministic Non-Periodic Flow," Journal of Atomsphere Science, Vol. 20, No. 2, June 1963, pp. 130-141.

[20] B. Li and W. S. Jiang, "Chaos Optimization Method and its Application," in Chinese, Control Theory and Applications, Vol. 14, No. 4, April 1997, pp. 613-615.

[21] L. Qiu, J. H. Tian, C. Q. Duan, X. N. Chen and Q. Huang, "The Application of Chaos Optimization Algorithm in Reservoir Optimal Operation," in Chinese, China Rural Water and Hydropower, Vol. 30, No. 7, July 2005, pp. 17-19.

[22] W. Liang, S. L. Chen, C. Y. He, D. Y. Liu and J. Rui, "Optimal Operation of Cascaded Hydropower Stations Based on Chaos Optimal Algorithm," in Chinese, Water Resources and Power, Vol. 26, No. 1, January 2008, pp. 63-66. 